\title{
Effects of Sunlight Exposure on the Quality Parameters of Bottled Water
}

\section{${ }^{1}$ AKHARAME, MO; ${ }^{1 *}$ EKHOSUEHI, A; ${ }^{2}$ OKOLAFOR, FI; ${ }^{1}$ SADOH, KA}

\author{
'Department of Environmental Management \& Toxicology, University of Benin, Benin City, Nigeria \\ ${ }^{2}$ Department of Science Laboratory Technology, University of Benin, Benin City, Nigeria
}

*Corresponding Author Email: amen.ekhosuehi@ uniben.edu

\begin{abstract}
This study was conducted to determine the physicochemical, heavy metal and microbiological effect of sunlight exposure on bottled water quality. Nine brands of bottled water commonly produced and sold in Benin City were exposed to sunlight for 0 day, 14 days and 28 days and evaluated for physicochemical parameters (pH, EC, TDS, alkalinity, hardness, $\left.\mathrm{Ca}^{2+}, \mathrm{Mg}^{2+}, \mathrm{Na}^{+}, \mathrm{K}^{+}, \mathrm{Cl}^{-}\right)$, heavy metals levels ( $\mathrm{Pb}, \mathrm{Cd}, \mathrm{Zn}, \mathrm{As}$ ) and microbial content using standard methods. Results obtained showed that $96.30 \%$ of the $\mathrm{pH}$ values were below the WHO (6.5-8.5), SON (6.5-8.5) and NIS (7.5) standards, a gradual decline in the values was also observed with increasing sunlight exposure. Other physicochemical parameters were within the established standards with steady increments in the values with increasing sunlight exposure, except for alkalinity which declined consistently due to increase in the acidity levels of the water samples. There was a general increase in the $\mathrm{Zn}$ concentration for all bottled water brands from 0 day to 28 days, except for brand $\mathrm{C}$ which remained the same, while $\mathrm{Pb}, \mathrm{Cd}$, As level were not detected. Interestingly, a gradual decrease of the microbial population (total coliform) of the bottled water with increasing exposure to sunlight was observed. In all, the varying exposure to sunlight did not result in statistical significant changes $(\mathrm{P}<0.05)$ in the water quality.
\end{abstract}

\section{DOI: https://dx.doi.org/10.4314/jasem.v22i5.31}

Copyright: Copyright () 2018 Akharame et al. This is an open access article distributed under the Creative Commons Attribution License (CCL), which permits unrestricted use, distribution, and reproduction in any medium, provided the original work is properly cited.

Dates: Received: 01 April 2018; Revised: 18 April: 2018; Accepted: 22 April 2018

Keywords: Bottled water, sunlight exposure, physicochemical parameters, heavy metals, microbial content.

Water is essential for life as it plays a key role in body metabolism and properfunctioning of cells (Kleiner, 1999). Drinking good quality water is vital for human health and development (UNICEF and WHO, 2012).A reliable supply of good drinking water is needed to promote healthy living amongst the inhabitants of any geological region (Isikwuel and Chikezie, 2014).Bottled water consumption has become very widespread and it appears to have filled the need for safe drinking water which has led to the tremendous increase in its demand. Common reasons for this spike in its consumption include factors such as taste, convenience or fashion, also issues bothering on safety and potential health benefits are considered (WHO, 2006).

In Nigeria, bottled water has become one of the highest consumable commodities for both the middle and high social class in urban areas. Hence, it is mandatory that bottled water brands are registered by the National Agency for Food and Drug Administration and Control (NAFDAC) which is the regulatory body responsible for monitoring and ensuring quality of bottled water produced and sold in the country. Sales of bottled water are high especially in major traffic channels where traders hawk various brands of bottled water on the road. Most restaurants and fast food outlets also sell bottled water. However, the method of storage before it is traded have become of increasing concern. Studies had shown that plastics bottles (polyethylene terephthalate) are susceptible to degradation under high temperature (Bach et al., 2009), and it is a common practice for local stores to leave bottled water exposed to sunlight before being traded. This investigation was carried out to determine the effect of sunlight exposure on the physicochemical properties, heavy metals levels and microbial content of some selected bottled water sold in Benin City and to underscore the possible health effect of drinking bottled water exposed to sunlight over a period of time.

\section{MATERIALS AND METHODS}

Study Area: The study was carried out in Benin City, Edo State, Nigeria. The City is located in the Southsouth region of Nigeria with an estimated average population of 3,206,531 in the 2006 general census (Annang, 2011). Benin City lies between latitudes $6^{0}$ $20^{\prime}$ and $6^{\circ} 58^{\prime}$ north and between longitudes $5^{0} 35$ and $6^{0} 41^{\prime}$ 'east of the Greenwich Meridian which is found within the sub-humid tropical region (Eseigbe and Magnus, 2012). It has an average temperature of about $27^{\circ} \mathrm{C}$ and an annual rainfall of over $2000 \mathrm{~mm}$ (Eseigbe et al., 2007). 
Sampling and analysis: Nine bottled water brands produced and sold in Benin City and environs were selected for this study. The bottled water were collected directly from production factories and subjected to 0,14 and 28 days exposure to sunlight. The water samples were exposed to $9 \mathrm{~h}$ sunlight per day between the hours of $8 \mathrm{am}$ to $5 \mathrm{pm}$. The physicochemical properties, heavy metals levels and microbial content of the first sets of samples were analyzed immediately after collection (control), while others were analyzed after exposure to sunlight for 14 days and 28 days, respectively. The physicochemical parameters investigated in this study were $\mathrm{pH}, \mathrm{EC}$, TDS, alkalinity, hardness, $\mathrm{Ca}^{2+}, \mathrm{Mg}^{2+}, \mathrm{Na}^{+}, \mathrm{K}^{+}$, and $\mathrm{Cl}^{-}$ , while the heavy metals were $\mathrm{Pb}, \mathrm{Cd}$, As and $\mathrm{Zn}$. Also, microbial assessment of the bottled water samples was investigated with the increasing sunlight exposure.

Microbiological Assessment: Sampled bottled water were serially diluted ( 2 folds) using $9 \mathrm{ml}$ of sterile distilled water against $1 \mathrm{mlof}$ the sample. Thereafter, $19 \mathrm{ml}$ of already prepared MacConkey and nutrient agar was poured into each of the petri-dishes and gently swirled so that the medium was evenly distributed in the petri dishes. The nutrient agar plates and MacConkey agar were both incubated at $35^{\circ} \mathrm{C}$ for $24 \mathrm{~h}$. The colonies of the nutrient agar and MacConkey agar plates were counted and expressed as colony forming units per $\mathrm{ml}\left(\mathrm{cfuml}^{-1}\right)$. Pure cultures of isolates were further obtained for characterization and identification according to standard procedures.

The results were described according to Bergeys' manual of determinative bacteriology (Breed et al., 1962). The colonial features of the fungal colonies were elucidated along with the morphological features of the fungi using compound microscope. The investigation of the morphological structures of fungi was done after being placed in lactophenol and cotton blue covered with cover slip. The fungal types were analyzed for each sampling exercise. The species were identified on the basis of micro and macro morphology and reverse and surface colouration of colonies grown on the Sabouraud dextrose agar media. The fungal isolates were identified using the methods described by Barnett and Hunter (1998).

Determination of heavy metals and physicochemical parameters of water samples: The atomic absorption spectrophotometry method was used for the rapid determination of heavy metals which included lead $(\mathrm{Pb})$, Cadmium (Cd), Arsenic (As) and Zinc ( $\mathrm{Zn})$ in the bottled water samples. Samples were not pretreated before analysis. The water samples were analyzed for their various metallic contents using Atomic Absorption Spectrophotometer, Unican 929 AA spectrometer (APHA 1998).
The AAS was also used to evaluate the levels of $\mathrm{Na}^{+}$ and $\mathrm{K}^{+}$. The $\mathrm{pH}$, TDS and EC were determined using Adwa multi-parameter meter (model AD8000) following standard procedures, while total hardness, $\mathrm{Mg}^{2+}$ and $\mathrm{Ca}^{2+}$ were measured by EDTA titrimetric method (APHA 1998). Alkalinity was determined by titrimetric method with methyl orange indicator. Nitrate and chlorine contents were analyzed using a spectrophotometer (HACH DR 3900 model).

Statistical analysis: Data was analyzed using SPSS statistical package and result presented in means of duplicate determinations.

\section{RESULTS AND DISCUSSION}

Total heterotrophic microbial counts of bottled water exposed to sunlight at 0,14 and 28 days revealed bacteria counts $1.0 \times 10^{2}$ to $9.0 \times 10^{2} \mathrm{cfu} / \mathrm{ml}$, coliform counts $2.0 \times 10^{3}$ to $8.0 \times 10^{3} \mathrm{cfu} / \mathrm{ml}$ and fungi counts $1.0 \times 10^{2}$ to $5.0 \times 10^{1} \mathrm{cfu} / \mathrm{ml}$ (Table 1 ). The result of total heterotrophic bacteria count showed a reduction in counts from 0 to 28 days exposure; coliform counts were recorded in few locations such as A, C, E, G and I. Total heterotrophic fungi count also showed slight decrease in counts from 0 to 28 days exposure. This result may be attributed to effect of solar energy in the decongestion/sterilization of microbial population of water (Dessie et al., 2014). Leclerc and Moreau (2002) reported that bottled water may contain high bacteria count as a result of natural biological process mainly from multiplication of these bacteria that were present in low number in the water source or from unhygienic bottling processes or equipment as opined by Georgieva and Dimitrova (2016). High heterotrophic bacteria plate counts are often directly linked to the effectiveness of water treatment processes (WHO, 2003). The result of this study, correlates with Lonnen et al. (2005), who recorded solar disinfection and solar photocatalytic disinfection, achieved at least a $4 \log$ unit reduction in viability against fungi species in drinking water. Meguiga (2009) posited that the counts for all tested microorganisms in water samples when exposed to sunlight decrease during storage period.

Staphylococcus aureus, Staphylococcus epidermidis, Azotobacter sp, Pseudomonas sp., Yeast, Penicillium italicum, Penicillium oxalicum, Mucor sp and Cladosporium sp were isolated from the water samples. Coliforms were not isolated asno growth was observed after exposure to sunlight. The results of microbial species isolated here, is in agreement with the study of Obiri et al. (2003), who reported that the coliforms die-off range was between $43-91 \%$ after $6 \mathrm{hrs}$ in sunlight and $51-100 \%$ after 48 hours at room temperature 
The physicochemical properties of the plastic bottled water after sunlightexposure are presented in Table 2, 3 and 4 . The $\mathrm{pH}$ value of water samples ranged from 4.8 to 6.5 (zero day exposure), 4.7 to 6.3 (14 days exposure) and 4.7 to 6.2 (28 days exposure). The trend showed that the $\mathrm{pH}$ of the water samples decreased gradually with increasing exposure to sunlight. Generally, the $\mathrm{pH}$ levelsrecorded did not meet the standard limit for drinking water (6.5 to 8.5) by WHO, SON and NIS, except for sample A on day zero. Water $\mathrm{pH}$ is majorly influenced by geology of catchment area and buffering capacityof water (Muhammad et al., 2011).Several documented researches report thatthe $\mathrm{pH}$ level of the underground water in Benin City is slightly acidic and was attributed to the geological formation and processes of the aquifer (Ezeigbo, 1988; Yasele and Idiata, 2012). This could be the cause of the low $\mathrm{pH}$ value of the water samples as the bottled water producersmay have failed to correct this defect. Total dissolved solids is a term used to describe the inorganic salt and small amount of organic matter present in water such as calcium,magnesium, sodium, potassium cation, and in addition carbonate, chloride, bicarbonate, sulfate, and nitrate (WHO, 1996;WHO, 2004). There was significant incrementin TDS values from 0 day to 14 days and 28 days exposure to sunlight. The TDS values range from $(5-122 \mathrm{mg} / \mathrm{l})$ for zero day, $(7.5-141 \mathrm{mg} / \mathrm{l})$ for 14 days and $(5.5-$ $139 \mathrm{mg} / \mathrm{l})$. The result of the TDS is in line with several reported investigations (Muhammad et al., 2011;
Toma, 2009; Tahir, 2010), and the values were within the standard limit set by WHO, SON and NIS.

The Electrical Conductivity (EC) of the bottled water exposed to sunlight showed significant increase for all samples, ranging from $(10-242 \mathrm{~ms} / \mathrm{cm})$ for 0 day exposure, $(16-284 \mathrm{~ms} / \mathrm{cm})$ for 14 days exposure and $(17-291 \mathrm{~ms} / \mathrm{cm})$ for 28 days exposure. This increase in EC may be occasioned by prolonged exposure to sunlight overtime, as the trend obtained was similar to the result obtained by Janan et al. (2013) and Muhammad et al. (2011) under similar conditions. The increase in EC and TDS which are interrelated also correlate with the increasing ions for other physicochemical parameters and heavy metal (zinc) with increase in sunlight exposure.

Alkalinity is the measure of water capacity to neutralize a strong acid. The mean level of alkalinity in the bottled water samples ranged from $(3.1-35.1$ $\mathrm{mg} / \mathrm{l})$ for zero day, $(3.1-29.0 \mathrm{mg} / \mathrm{l})$ for 14 days and $(3.1-25.9 \mathrm{mg} / \mathrm{l})$ for 28 days. The alkalinity values revealed significant reduction from day zero to 14 days and finally to 28 days period. Hardness results from metal cations such as calcium and magnesium. All divalent and polyvalent cations cause hardness when they react to certain anions such as carbonateand sulfate to form a precipitate (Abd El-Salam et al., 2008).

\begin{tabular}{|c|c|c|c|c|}
\hline $\begin{array}{l}\text { Sample } \\
\text { Code }\end{array}$ & $\begin{array}{l}\text { Days of } \\
\text { Exposure to } \\
\text { sun light }\end{array}$ & $\begin{array}{l}\text { Total heterotrophic } \\
\text { bacteria count } \\
(\mathrm{cfu} / \mathrm{ml})\end{array}$ & $\begin{array}{l}\text { Total Coliform } \\
\text { count (cfu/ml) }\end{array}$ & $\begin{array}{l}\text { Total heterotrophic } \\
\text { fungi count } \\
\text { (cfu/ml) }\end{array}$ \\
\hline \multirow{3}{*}{ A } & 0 & $6.0 \times 10^{3}$ & $5.0 \times 10^{1}$ & $5.0 \times 10^{1}$ \\
\hline & 14 & $4.0 \times 10^{1}$ & NG & $4.0 \times 10^{1}$ \\
\hline & 28 & $2.0 \times 10^{1}$ & NG & $4.0 \times 10^{1}$ \\
\hline \multirow{3}{*}{ B } & 0 & $2.0 \times 10^{4}$ & NG & $1.0 \times 10^{1}$ \\
\hline & 14 & $2.0 \times 10^{3}$ & NG & $1.0 \times 10^{1}$ \\
\hline & 28 & $4.0 \times 10^{2}$ & NG & NG \\
\hline \multirow{3}{*}{$\mathrm{C}$} & 0 & $1.0 \times 10^{3}$ & $8.0 \times 10^{1}$ & $2.0 \times 10^{1}$ \\
\hline & 14 & NG & NG & $1.0 \times 10^{1}$ \\
\hline & 28 & NG & NG & NG \\
\hline \multirow{3}{*}{$\mathrm{D}$} & 0 & $6.0 \times 10^{2}$ & NG & $3.0 \times 10^{1}$ \\
\hline & 14 & NG & NG & $2.0 \times 10^{1}$ \\
\hline & 28 & $4.0 \times 10^{1}$ & NG & NG \\
\hline \multirow{3}{*}{$\mathrm{E}$} & 0 & $3.0 \times 10^{1}$ & $2.0 \times 10^{3}$ & NG \\
\hline & 14 & $2.0 \times 10^{3}$ & NG & $1.0 \times 10^{1}$ \\
\hline & 28 & $4.0 \times 10^{3}$ & NG & NG \\
\hline \multirow{3}{*}{$\mathrm{F}$} & 0 & $1.0 \times 10^{1}$ & NG & $3.0 \times 10^{1}$ \\
\hline & 14 & $4.0 \times 10^{2}$ & NG & $2.0 \times 10^{1}$ \\
\hline & 28 & $3.0 \times 10^{2}$ & NG & NG \\
\hline \multirow{3}{*}{ G } & 0 & $1.0 \times 10^{2}$ & NG & $3.0 \times 10^{1}$ \\
\hline & 14 & $1.0 \times 10^{2}$ & NG & $3.0 \times 10^{1}$ \\
\hline & 28 & NG & NG & NG \\
\hline \multirow{3}{*}{$\mathrm{H}$} & 0 & $8.0 \times 10^{3}$ & $5.0 \times 10^{-1}$ & $3.0 \times 10^{1}$ \\
\hline & 14 & NG & NG & $2.0 \times 10^{1}$ \\
\hline & 28 & $5.0 \times 10^{-1}$ & NG & NG \\
\hline \multirow{3}{*}{ I } & 0 & $9.0 \times 10^{2}$ & $3.0 \times 10^{1}$ & $1.0 \times 10^{2}$ \\
\hline & 14 & NG & NG & $5.0 \times 10^{1}$ \\
\hline & 28 & $2.0 \times 10^{2}$ & NG & $1.0 \times 10^{1}$ \\
\hline
\end{tabular}


Table 2: Physiochemical study of water at zero day exposure to sun light

\begin{tabular}{|c|c|c|c|c|c|c|c|c|c|c|c|c|}
\hline \multirow[t]{2}{*}{ Parameters } & \multicolumn{9}{|l|}{ Sample ID } & \multicolumn{3}{|c|}{ Established standards } \\
\hline & $\mathbf{A}$ & B & $\mathbf{C}$ & $\begin{array}{l}\mathbf{D} \\
\end{array}$ & $\mathbf{E}$ & $\mathbf{F}$ & G & $\mathbf{H}$ & $\mathbf{I}$ & WHO & SON & NIS \\
\hline $\mathrm{pH}$ & $6.5 \pm 0.07$ & $5.9 \pm 0.14$ & $5.3 \pm 0.14$ & $5.1 \pm 0.00$ & $4.8 \pm 0.00$ & $6.0 \pm 0.07$ & $5.8 \pm 0.07$ & $6.1 \pm 0.14$ & $5.7 \pm 0.14$ & $\begin{array}{l}6.5- \\
8.5\end{array}$ & $\begin{array}{l}6.5- \\
8.5\end{array}$ & 7.50 \\
\hline EC & $110 \pm 1.41$ & $131 \pm 1.41$ & $23 \pm 0.00$ & $21 \pm 3.54$ & $242 \pm 0.00$ & $80 \pm 0.00$ & $44 \pm 1.41$ & $17 \pm 1.41$ & $10 \pm 0.71$ & 1500 & 1000 & 1000 \\
\hline TDS & $56 \pm 1.41$ & $65 \pm 1.41$ & $12 \pm 0.00$ & $10 \pm 1.41$ & $122 \pm 0.00$ & $40 \pm 0.71$ & $22 \pm 0.71$ & $9 \pm 0.00$ & $5 \pm 0.00$ & $\begin{array}{l}600- \\
1000\end{array}$ & 500 & 500 \\
\hline Alkalinity & $35.1 \pm 2.16$ & $3.1 \pm 0.00$ & $6.1 \pm 0.00$ & $4.58 \pm 2.16$ & $3.1 \pm 0.00$ & $25.9 \pm 2.16$ & $6.1 \pm 0.00$ & $3.1 \pm 0.00$ & $6.1 \pm 0.00$ & NS & NS & NS \\
\hline Hardness & $10.4 \pm 1.12$ & $24.8 \pm 1.13$ & $12.0 \pm 3.39$ & $15.2 \pm 1.13$ & $37.6 \pm 3.39$ & $4.8 \pm 0.00$ & $2.4 \pm 1.13$ & $5.6 \pm 1.13$ & $3.2 \pm 0.00$ & 100 & 100 & 100 \\
\hline $\mathrm{Ca}^{+2}$ & $2.25 \pm 0.46$ & $3.85 \pm 0$ & $3.85 \pm 0.9$ & $3.85 \pm 0.0$ & $9.31 \pm 1.3$ & $1.28 \pm 0.00$ & $0.64 \pm 0.0$ & $1.60 \pm 0.4$ & $0.64 \pm 0.0$ & 75 & NS & 50 \\
\hline $\mathrm{Mg}^{2+}$ & $1.17 \pm 0.00$ & $3.70 \pm 0.28$ & $0.59 \pm 0.28$ & $1.37 \pm 0.28$ & $3.50 \pm 1.65$ & $0.39 \pm 0.00$ & $0.20 \pm 0.28$ & $0.39 \pm 0.00$ & $0.39 \pm 0.00$ & 30 & 0.2 & 0.2 \\
\hline $\mathrm{Na}$ & $4.03 \pm 0.06$ & $13.0 \pm 0.08$ & $1.37 \pm 0.23$ & $0.91 \pm 0.16$ & $24.0 \pm 0.19$ & $1.96 \pm 0.06$ & $1.29 \pm 0.35$ & $1.08 \pm 0.13$ & $0.62 \pm 0.02$ & 200 & 200 & 200 \\
\hline $\mathrm{K}^{+}$ & $3.0 \pm 0.18$ & $10.1 \pm 0.00$ & $0.64 \pm 0.41$ & $0.45 \pm 0.13$ & $18.7 \pm 0.00$ & $0.13 \pm 0.007$ & $0.13 \pm 0$ & $0.1 \pm 0.00$ & $0.07 \pm 0.0$ & NS & NS & NS \\
\hline $\mathrm{Cl}^{-}$ & $14.2 \pm 0.0$ & $39.0 \pm 5.0$ & $7.09 \pm 0.0$ & $7.09 \pm 0.0$ & $60.3 \pm 5.0$ & $7.09 \pm 0.00$ & $17.7 \pm 5.0$ & $14.2 \pm 0.0$ & $7.09 \pm 0.0$ & 250 & 25.0 & 100 \\
\hline
\end{tabular}

$W H O=$ World Health Organization, SON= Standard Organization of Nigeria, NIS= Nigerian Industrial Standards

Table 3: Physicochemical study of water after 14 days exposure to sunlight:

\begin{tabular}{|c|c|c|c|c|c|c|c|c|c|c|c|c|}
\hline \multirow[t]{2}{*}{ Parsmetern } & \multicolumn{9}{|l|}{ Sample ID } & \multicolumn{3}{|c|}{ Established standards } \\
\hline & $A$ & B & $\mathrm{C}$ & D & E & F & $\mathrm{G}$ & $\mathrm{H}$ & I & WHO & SON & NIS \\
\hline $\mathrm{pH}$ & $6.2 \pm 0$ & $5.45 \pm 0.21$ & $5.65=0.07$ & $5.35 \pm 0.21$ & $4.7 \pm 0.14$ & $5.95 \pm 0.07$ & $5.7 \pm 0.14$ & $5.5 \pm 0.14$ & $5.2 \pm 0.14$ & $\begin{array}{l}6.5- \\
3.5\end{array}$ & $\begin{array}{l}6.5- \\
8.5\end{array}$ & 7.50 \\
\hline $\mathrm{EC}$ & $116 \pm 0$ & $151 \pm 0$ & $54 \pm 2.83$ & $32 \pm 2.83$ & $291 \pm 0$ & $83 \pm 1.41$ & $44 \pm 2.83$ & $17.5 \pm 0.71$ & $17 \pm 1.41$ & 1500 & 1000 & 1000 \\
\hline TDS & $58 \pm 0$ & $75 \pm 0$ & $27=1.41$ & $16 \pm 1.41$ & $139 \pm 0$ & $41.5=0.7$ & $22=1.41$ & $8.5 \pm 0.71$ & $5.5 \pm 0.71$ & $\begin{array}{l}600- \\
1000\end{array}$ & 500 & 500 \\
\hline Alkalinity & $25.9=2.1$ & $3.05 \pm 0$ & $12.2 \pm 0$ & $7.63 \pm 2.1$ & $3.05 \pm 0$ & $22.9=2.1$ & $4.58=2.1$ & $3.05 \pm 0$ & $4.58=2.1$ & NIL & NIL & NIL \\
\hline Hardus: & $3.01 \pm 0$ & $17.6 \pm 2.2$ & $17.6 \pm 0$ & $18.9=1.8$ & $42.4 \pm 1.1$ & $7.21 \pm 1.1$ & $4 \pm 1.13$ & $3.01 \pm 0$ & $6.41 \pm 0$ & 100 & 100 & 100 \\
\hline $\mathrm{Cz}^{2-}$ & $1.6 \pm 0.45$ & $3.35 \pm 0$ & $5.77 \pm 0$ & $3.53=0.4$ & $8.34 \pm 0.9$ & $1.6 \pm 0.45$ & $0.96 \pm 0.4$ & $1.92=0$ & $1.6 \pm 0.45$ & 75 & NIL & 30 \\
\hline $\mathrm{Mg}^{3+}$ & $0.98=0.2$ & $1.95=0.5$ & $1.75=1.3$ & $4.12=2.4$ & $2.73=2.7$ & $0.59=0.2$ & $0.59 \pm 0.2$ & $0.73 \pm 0$ & $0.59 \pm 0.2$ & 30 & 0.2 & 0.2 \\
\hline $\mathrm{Na}$ & $3.19=0.003$ & $3.77=0.006$ & $3.45=0.003$ & $1.15=0.003$ & $3.77 \pm 0.006$ & $3.71=0.004$ & $3.65=0.002$ & $2.89=0$ & $1.63 \pm 0.52$ & 200 & 200 & 200 \\
\hline $\mathrm{K}^{*}$ & $0.21=0.0$ & $4.43 \pm 0.0$ & $0.76 \pm 0$ & $0.15 \pm 0$ & $6.51 \pm 0.0$ & $0.87 \pm 0$ & $0.26 \pm 0$ & $0.29=0.0$ & $0.34 \pm 0$ & NIL & $\mathrm{NIL}$ & NIL \\
\hline $\mathrm{Cl}$ & $10.6 \pm 5.0$ & $28.4 \pm 0$ & $10.6 \pm 5.0$ & $7.09=0$ & $53.2 \pm 5.0$ & $7.09=0$ & $14.2 \pm 0$ & $7.09 \pm 0$ & $7.09 \neq 0$ & 250 & 250 & 100 \\
\hline
\end{tabular}

All values are expressed in $\mathrm{m} / \mathrm{l}$, except electrical conductivity $(\mu \mathrm{S} / \mathrm{cm})$ and $\mathrm{pH}$ (no unit). Result expressed in Mean \pm Standard deviation, $N S=N o t$ stated, WHO $=$ World Health Organization, SON= Standard Organization of Nigeria, NIS= Nigerian Industrial Standards Table 4: Physiochemical study of water after 28 days exposure to sunlight

\begin{tabular}{|c|c|c|c|c|c|c|c|c|c|c|c|c|}
\hline \multirow[t]{2}{*}{ Parsmetern } & \multicolumn{9}{|l|}{ Sample ID } & \multicolumn{3}{|c|}{ Established staudards } \\
\hline & $A$ & B & $\mathrm{C}$ & D & $E$ & $\mathrm{~F}$ & G & $\mathrm{H}$ & I & WHO & SON & NIS \\
\hline $\mathrm{pH}$ & $6.3 \pm 0.07$ & $5.8 \pm 0.21$ & $5.7 \pm 0.00$ & $5.6 \pm 0.00$ & $4.7 \pm 0.07$ & $6.2 \pm 0.00$ & $5.5 \pm 0.07$ & $5.9 \pm 0.07$ & $5.6 \pm 0.21$ & $6.5-8.5$ & $\begin{array}{l}6.5- \\
8.5\end{array}$ & 7.50 \\
\hline $\mathrm{EC}$ & $117 \pm 0.71$ & $139 \pm 0.71$ & $41 \pm 14.1$ & $29=0.71$ & $284 \pm 2.12$ & $96 \neq 0.71$ & $48 \pm 4.94$ & $19 \pm 0.00$ & $16 \pm 2.12$ & 1500 & 1000 & 1000 \\
\hline $\mathrm{TDS}$ & $57.5 \pm 0.71$ & $68.5 \pm 0.71$ & $20=7.07$ & $14 \pm 0.00$ & $141.5=0.71$ & $42.5 \pm 0.71$ & $23.5=2.12$ & $9 \pm 0.00$ & $7.5 \pm 0.71$ & $\begin{array}{l}600 \\
1000\end{array}$ & 500 & 500 \\
\hline Allalimity & $29.0 \pm 2.16$ & $4.59 \pm 2.16$ & $10.7 \pm 6.47$ & $6.1 \pm 0.00$ & $3.05 \pm 0.00$ & $22.9 \pm 2.16$ & $3.05 \pm 0.00$ & $3.05 \pm 0.00$ & $4.58 \pm 2.16$ & NS & NS & NS \\
\hline Hardings: & $9.61=2.26$ & $20.0=1.13$ & $13.6 \pm 3.39$ & $16.8 \pm 1.13$ & $39.2 \pm 1.13$ & $4.8 \pm 0.00$ & $3.2 \pm 0.00$ & $4 \pm 1.13$ & $4=1.13$ & 100 & 100 & 100 \\
\hline $\mathrm{Cz}_{2=+}$ & $2.25 \pm 0.46$ & $4.17=0.45$ & $4.49 \pm 0.91$ & $2.57 \pm 0.00$ & $7.7 \pm 0.00$ & $0.96 \pm 0.45$ & $0.64=0.00$ & $1.28=0.00$ & $0.64 \pm 0$ & 75 & NS & 30 \\
\hline $\mathrm{Mg}^{3+}$ & $1.26 \pm 0.70$ & $2.33=0.00$ & $0.59 \pm 0.28$ & $2.53 \pm 0.28$ & $4.87=0.28$ & $0.59=0.28$ & $0.39 \pm 0.00$ & $0.20 \pm 0.28$ & $0.59=0.28$ & 30 & 0.2 & 0.2 \\
\hline $\mathrm{Na}$ & $7.93=0.04$ & $7.89 \pm 0.05$ & $4.51 \pm 1.97$ & $0.93 \pm 0.19$ & $7.91=0.02$ & $7.06 \pm 0.09$ & $7.92=0.005$ & $3.73 \pm 0.75$ & $1.94 \pm 0.09$ & 200 & 200 & 200 \\
\hline $\mathrm{X}^{*}$ & $1.57 \pm 0.03$ & $4.73=0.09$ & $0.21 \pm 0.04$ & $0.03 \pm 0.04$ & $9.82=0.13$ & $0.11=0.04$ & $0.16 \neq 0.04$ & $0.26 \pm 0.04$ & $0.21=0.04$ & NS & NS & NS \\
\hline $\mathrm{Cl}$ & $10.6 \pm 5.01$ & $31.9 \pm 5.01$ & $7.09 \pm 0.00$ & $7.09 \pm 0.00$ & $53.2 \pm 5.01$ & $7.09 \pm 0.00$ & $14.2 \pm 0.00$ & $10.6 \pm 5.01$ & $7.09 \pm 0$ & 250 & 25.0 & 100 \\
\hline
\end{tabular}

All values are expressed in $\mathrm{m} / \mathrm{l}$, except electrical conductivity $(\mu \mathrm{S} / \mathrm{cm})$ and $\mathrm{pH}$ (no unit). Result expressed in Mean \pm Standard deviation, NM $=$ Not mentioned $W H O=$ World Health Organization, SON= Standard Organization of Nigeria, NIS = Nigerian Industrial Standards

\begin{tabular}{|c|c|c|c|c|c|c|c|c|c|c|c|c|}
\hline \multirow[b]{2}{*}{$\begin{array}{l}\text { Sample } \\
\text { ID }\end{array}$} & \multicolumn{4}{|l|}{ Day zero } & \multicolumn{4}{|c|}{14 days sunlight exposure } & \multicolumn{4}{|c|}{28 days sunlight exposure } \\
\hline & $\overline{Z n}$ & $\mathrm{pb}$ & $\mathrm{Cd}$ & As & $\mathrm{Zn}$ & $\mathrm{Bb}$ & $\mathrm{Cd}$ & A5 & $\mathrm{Zn}$ & $\mathrm{pb}$ & $\mathrm{Cd}$ & A5 \\
\hline $\begin{array}{l}\mathrm{A} \\
\mathrm{B}\end{array}$ & $\begin{array}{l}0.025 \pm 0.007 \\
0.015 \pm 0.007\end{array}$ & $\begin{array}{l}<\mathrm{DL} \\
<\mathrm{DL}\end{array}$ & $\begin{array}{l}<D L \\
<D L\end{array}$ & $\begin{array}{l}<\mathrm{DL} \\
<\mathrm{DL}\end{array}$ & $\begin{array}{l}0.03 \pm 0.00 \\
0.015 \pm 0.007\end{array}$ & $\begin{array}{l}<\mathrm{DL} \\
<\mathrm{DL}\end{array}$ & $\begin{array}{l}<D L \\
<D L\end{array}$ & $\begin{array}{l}<\mathrm{DL} \\
<\mathrm{DL}\end{array}$ & $\begin{array}{l}0.03 \pm 0.00 \\
0.025 \pm 0.007\end{array}$ & $\begin{array}{l}<\mathrm{DL} \\
<\mathrm{DL}\end{array}$ & $\begin{array}{l}<\mathrm{DL} \\
<\mathrm{DL}\end{array}$ & $\begin{array}{l}<D L \\
<D L\end{array}$ \\
\hline $\mathrm{C}$ & $0.03 \pm 0.00$ & $<D L$ & $<D L$ & $<D L$ & $0.025 \pm 0.007$ & $<\mathrm{DL}$ & $<D L$ & $<D L$ & $0.03 \pm 0.00$ & $<\mathrm{DL}$ & $<D L$ & $<D L$ \\
\hline $\begin{array}{l}\mathrm{D} \\
\mathrm{E}\end{array}$ & $\begin{array}{l}0.015 \pm 0.007 \\
0.015 \pm 0.007\end{array}$ & $\begin{array}{l}<\mathrm{DL} \\
<\mathrm{DL}\end{array}$ & $\begin{array}{l}<D L \\
<D L\end{array}$ & $\begin{array}{l}<D L \\
<D L\end{array}$ & $\begin{array}{l}0.02 \pm 0.00 \\
0.025 \pm 0.007\end{array}$ & $\begin{array}{l}<\mathrm{DL} \\
<\mathrm{DL}\end{array}$ & $\begin{array}{l}<\mathrm{DL} \\
<\mathrm{DL}\end{array}$ & $\begin{array}{l}<\mathrm{DL} \\
<\mathrm{DL}\end{array}$ & $\begin{array}{l}0.025 \pm 0.007 \\
0.03 \pm 0.014\end{array}$ & $\begin{array}{l}<\mathrm{DL} \\
<\mathrm{DL}\end{array}$ & $\begin{array}{l}<\mathrm{DL} \\
<\mathrm{DL}\end{array}$ & $\begin{array}{l}<D L \\
<D L\end{array}$ \\
\hline $\mathrm{F}$ & $0.02 \pm 0.14$ & $<\mathrm{DL}$ & $<\mathrm{DL}$ & $<D L$ & $0.02 \pm 0.00$ & $<D L$ & $<D L$ & $<D L$ & $0.025 \pm 0.007$ & $<\mathrm{DL}$ & $<D L$ & $<D L$ \\
\hline G & $<D L$ & $<\mathrm{DL}$ & $<D L$ & $<\mathrm{DL}$ & $0.015 \pm 0.007$ & $<\mathrm{DL}$ & $<\mathrm{DL}$ & $<D L$ & $0.015 \pm 0.007$ & $<\mathrm{DL}$ & $<\mathrm{DL}$ & $<D L$ \\
\hline $\begin{array}{l}\mathrm{H} \\
\mathrm{I}\end{array}$ & $\begin{array}{l}0.02 \pm 0.014 \\
0.02 \pm 0.00\end{array}$ & $\begin{array}{l}<\mathrm{DL} \\
<\mathrm{DL}\end{array}$ & $\begin{array}{l}<D L \\
<D L\end{array}$ & $\begin{array}{l}<D L \\
<D L\end{array}$ & $\begin{array}{l}0.015 \pm 0.007 \\
0.02 \pm 0.00\end{array}$ & $\begin{array}{l}<\mathrm{DL} \\
<\mathrm{DL}\end{array}$ & $\begin{array}{l}<\mathrm{DL} \\
<\mathrm{DL}\end{array}$ & $\begin{array}{l}<\mathrm{DL} \\
<\mathrm{DL}\end{array}$ & $\begin{array}{l}0.025 \pm 0.007 \\
0.025 \pm 0.007\end{array}$ & $\begin{array}{l}<\mathrm{DL} \\
<\mathrm{DL}\end{array}$ & $\begin{array}{l}<\mathrm{DL} \\
<\mathrm{DL}\end{array}$ & $\begin{array}{l}<\mathrm{DL} \\
<\mathrm{DL}\end{array}$ \\
\hline Average & 0.016 & - & - & - & 0.021 & - & - & - & 0.026 & - & - & - \\
\hline
\end{tabular}

Heavy metal levels are expressed in $\mathrm{mg} / \mathrm{l} . \angle D L=$ below detection limit; detection limit for $\mathrm{Zn}=0.01, \mathrm{~Pb}=0.05, \mathrm{Cd}=0.005 \mathrm{and} \mathrm{As}=0.01 \mathrm{mg} / \mathrm{l}$

The mean hardness values for day 0,14 and 28 ranged from $(3.2-42.4 \mathrm{mg} / \mathrm{l})$, significant increase in hardness was recorded but the values were within the standard limits. Calcium and magnesium are two of many inorganic substances responsible for creating hardness in water (Kenneth et al., 2003). They are regarded as important major cations in water. Calcium and magnesium concentration in the sampled bottled water varied from $\left(\mathrm{Ca}: 0.6-2.85 \mathrm{mg} / \mathrm{l} ; \mathrm{Mg}^{2+}: 0.20-3.70\right.$ $\mathrm{mg} / \mathrm{l})$; (Ca: $0.64-4.49 \mathrm{mg} / \mathrm{l} ; \mathrm{Mg}^{2+}: 0.20$ - $\left.4.87 \mathrm{mg} / \mathrm{l}\right)$,
(Ca: 0.96 -8.34; $\mathrm{Mg}^{2+}$ : 0.59 -4.12 mg/l) for 0 day, 14 days and 28 days exposure to sunlight, respectively. The values were within the WHO standard. The observed fluctuating physicochemical parameters of bottled water under sunlight can possibly be attributed to degradation of plastic bottle due to different temperature, resistance and sensitivity (Muhammad et al., 2011). 
For the heavy metals assessment, the levels of $\mathrm{Pb}, \mathrm{Cd}$ and As were found to be below the detection limit of $0.05,0.005$ and $0.1 \mathrm{mg} / 1$, respectively of the equipment used for the analysis. The $\mathrm{Zn}$ levels in all the bottled water samples were within stipulated limits set by WHO and NIS, and the exposure to sunlight did not yield significant increase in the concentrations. Only slight increase in the weighted averages for the various exposures showed marginal increment from 0.016 to 0.021 and $0.026 \mathrm{mg} / \mathrm{l}$ for zero, 14 and 28 day exposure to sunlight, respectively.

Conclusions: From the forgoing, it was observed that the $\mathrm{pH}$ of all the bottled water analyzed except for brand $\mathrm{A}$ at zero day, were below recommended standards by WHO, SON and NIS. All other physiochemical variables analyzed were well within the established standards. However, increment in levels were observed in all the physicochemical parameters analyzed in each brand after varying sunlight exposure, except for alkalinity that decreased with increasing acidity of the water samples. Also, a gradual decrease of the microbial population in the water samples with increasing exposure to sunlight was observed, which denotes the effect of solar disinfection. In all, the changes observed after varying exposure to sunlight was not significant $(\mathrm{P}<0.05)$. However, it is advisable to store bottled water properly prior to being sold to consumers.

\section{REFERENCES}

Abd El-Salam, MAA; El-Ghitany, EMA; Kassem, MMM (2008). Quality of bottled water brands in Egypt. J. Egypt Public Health Assoc. 83(5 \& 6): 370-39

American Public Health Association (1998).Standard Methods for the Examination of Water and Waste Water.20 ${ }^{\text {th }}$ ed. Washington, DC, USA.p.1220.

Annang, A (2011). Nigeria census in the midst of politics and ethnicity: Where do we go from here?. Afr.. J. Cultr. Philo. Soc. 1(1): 12-19.

Bach, C; Dauchy, X; Etienne, S (2009). Characterization of poly (ethylene terephthalate) used in commercial bottled water. Mater. Sci. Eng. 5(1): 5-10.

Barnett, HL; Hunter, BB (1998).Illustrated Genera of Imperfect Fungi. 4th Edition, APS Press, St. Paul, p. 218

Breed, SR; Murray, EGD; Smith, RN (1962). Bergey's Manual of Determinative Bacteriology. Baltimore, Md: Williams and Wilkins, p.1094
Dessie, A; Alemayehu, E; Mekonen, S; Leggesse, W; Woos, H; Ambelu, A (2014). Solar disinfection: An appropriate approach for low-cost household water treatment technology in Southwestern Ethiopia. J. Environ. Health Sci. Eng. 12: 25-30

Eseigbe, JO; Magnus, OO (2012).Aspect of gully erosion in Benin City, Edo State, Nigeria. Res. Humanities Soc. Sci. 2 (7): 21-26.

Eseigbe, JO; Omonfonmwan, SI; Kadiri, MA (2007).Solid waste generation and management in Benin Metropolis. Confluence J. Environ. Stud. 2 (2): 34-44.

Ezeigbo, HI (1988). Geological and hydrological influence on the Nigerian environment. J. Water Resour. 2: 36-44

Georgieva, V; Dimitrova, Y (2016).Study of the microbiological quality of Bulgarian bottled water in terms of its contamination with Pseudomonas aeruginosa. Cent. Eur. J. Public Health 24: 326330

Isikwuel, MO; Chikezie, A (2014). Quality assessment of various sachet water brands marketed in Bauchi Metropolis of Nigeria. Inter. J. Advanc. Eng. Technol. 6: 2489-2495.

Janan, JT; Nihal, SH; Bakhtiar, AM (2012). Effect of storage condition on some bottled water quality in Erbil City, Kurdistan Region-Iraq. Inter. J. Software Web Sci. 3 (1): 33-43

Kenneth, MV (2003). Clean water: An Introduction to Water Quality and Water Pollution Control.2nd Edition. First OSU Press. USA.

Kleiner, SM (1999). Water: An essential but overlooked nutrient. J. Am Diet Assoc., 99(2): 200-206

Leclerc, H; Moreau, A (2002). Microbiological safety of natural mineral water. FEMS Microbiol. Rev.26: 207-222

Lonnen, J; Kilvington, S; Kehoe, S; Al-Touati, F; McGuigan, K (2005). Solar and photocatalytic disinfection of protozoan, fungal and bacterial microbes in drinking water. Wat. Res. 39:877-883

Meguigan, KG; Joyce, TM; Elmoore, MM; Conroy, RM (1996).Inactivating of faecal bacteria in drinking water by solar heating. Appl. Environ. Microbiol. 62 (2): 399 - 402. 
Muhammad, SG; Esmail, LS; Hasan, SH (2011).Effect of storage temperature and sunlight exposure on the physicochemical properties of bottled water in Kurdistan region-Iraq.J. Appl. Sci. Environ. Manage. 15(1): 147-154

Nigerian Industrial Standard (NIS), (2007).Nigerian Standard for Drinking Water Quality. ISC 13.060.20 http://www.unicef.org/nigeria/ngpublication.

Obiri-Daso, K; Okore-Hanson, A; Jones, K (2003). The microbiological quality of drinking water sold on the streets in Kumasi, Ghana. Lett. Appl. Microbial. 37(4): 334-339

SON (2007).Nigerian Standards for Drinking Water Quality. Nigerian Industrial Std, NIS 554, Published by the Standard Organization of Nigeria, 13/14 V.A. Street off Admiralty way, Lekki, Lagos Nigeria.

Tahir, LO (2010).Study on Quality Assessment of Some Available Bottled Drinking Water within Hawler Markets. M.Sc. thesis. University of Salahaddin, College of Science, 2010.

Toma, JJ (2009). Study of water quality of various trading marks of potable bottled water in Erbil City, Iraq.J. Pure Appl. Sci. 22(2): 1-11
UNICEF \& World Health Organization. (2012). Progress on Drinking Water and Sanitation: 2012 Update. New York: WHO/UNICEF Joint Monitoring Programme for Water Supply and Sanitation.

World Health Organization (1996).Guidelines for Drinking-water Quality- Health Criteria and Other Supporting Information. Vol. 1: 2nd ed. Geneva, Switzerland

World Health Organization (2004).Guidelines for Drinking-water Quality- Recommendations. Vol. 1: 3rd ed. Geneva, Switzerland

World Health Organization (2003). Heterotrophic Plate Count and Drinking-water Safety. Bartram, J; Controvo, J; Exner, M; Fricker, C; Glasmacher, A (Eds). IWA Publishing, London

World Health Organization (2006).Protecting Groundwater for Health: Managing the Quality of Drinking Water Sources. IWA Publishing, London

Yasele, JU; Idiata, DJ (2012).Determining the borehole water quality in Edo South and Edo North areas of Edo State. Res. J. Eng. Appl. Sci. 1 (4): 209-213 